\title{
Characterization of Retinal Disease Progression in a 1-Year Longitudinal Study of Eyes With Mild Nonproliferative Retinopathy in Diabetes Type 2
}

\author{
Luisa Ribeiro, ${ }^{1}$ Francesco Bandello, ${ }^{2}$ Amparo Navea Tejerina, ${ }^{3}$ Stela Vujosevic, ${ }^{4}$ Monica Varano, ${ }^{5}$ \\ Catherine Egan, ${ }^{6}$ Sobha Sivaprasad, ${ }^{7}$ Geeta Menon, ${ }^{8}$ Pascale Massin, ${ }^{9}$ Frank D. Verbraak, ${ }^{10}$ \\ Henrik Lund-Andersen, ${ }^{11}$ Jose P. Martinez, ${ }^{12}$ Ignasi Jürgens, ${ }^{13}$ Erica Smets, ${ }^{14}$ Caroline Coriat, ${ }^{15}$ \\ Peter Wiedemann, ${ }^{16}$ Victor Ágoas, ${ }^{17}$ Giuseppe Querques, ${ }^{18}$ Frank G. Holz, ${ }^{19}$ Sandrina Nunes, ${ }^{1}$ \\ Catarina Neves, ${ }^{1}$ and José Cunha-Vaz; ${ }^{1}$ for the EVICR.net Study Group
}

\footnotetext{
${ }^{1}$ Association for Innovation and Biomedical Research on Light and Image (AIBILI), Coimbra, Portugal

${ }^{2}$ Department of Ophthalmology, University Vita Salute-Scientific Institute of San Raffael, Milan, Italy

${ }^{3}$ Fundación para la Investigacion Biomedica y Sanitaria, FISABIO-OFTALMOLOGIA, Valencia, Spain

${ }^{4}$ Centre for Clinical Trials, Department of Ophthalmology, University of Padova, Padova, Italy

${ }^{5}$ G.B. Bietti Eye Foundation-IRCCS, Rome, Italy

${ }^{6}$ Clinical Trials Unit, Moorfields Eye Hospital, NHS Foundation Trust, London, United Kingdom

${ }^{7}$ Laser and Retinal Research Unit, King's Health Partners, London, United Kingdom

${ }^{8}$ Ophthalmology Clinical Trials Unit, Frimley Park Hospital Foundation Trust, Frimley, United Kingdom

${ }^{9}$ Department of Ophthalmology, Lariboisière Hospital, Paris, France

${ }^{10}$ Department of Ophthalmology, Academic Medical Center, Amsterdam, The Netherlands

${ }^{11}$ Department of Ophthalmology, Glostrup Hospital, Copenhagen University, Glostrup, Denmark

${ }^{12}$ Rotterdam Eye Hospital, Rotterdam, The Netherlands

${ }^{13}$ Institut Català de Retina (ICR), Barcelona, Spain

${ }^{14}$ Antwerp University Hospital, Department of Ophthalmology, Antwerp, Belgium

${ }^{15}$ Centre d'Investigation Clinique, Centre National d'Ophtalmologie des Quinze-Vingts, Paris, France

${ }^{16}$ University Eye Hospital Leipzig, Leipzig, Germany

${ }^{17}$ Instituto de Oftalmologia Dr. Gama Pinto, Lisboa, Portugal

${ }^{18}$ Centre Hospitalier Intercommunal de Creteil, University Paris-Est Creteil, Creteil, France

${ }^{19}$ Department of Ophthalmology, University of Bonn, Bonn, Germany
}

Correspondence: José Cunha-Vaz, AIBILI, Azinhaga de Santa Comba, Celas, 3000-548, Coimbra, Portugal; cunhavaz@aibili.pt.

Submitted: February 18, 2015

Accepted: July 14, 2015

Citation: Ribeiro L, Bandello F, Tejerina AN, et al.; for the EVICR.net Study Group. Characterization of retinal disease progression in a 1-year longitudinal study of eyes with mild nonproliferative retinopathy in diabetes type 2. Invest Ophthalmol Vis Sci. 2015;56:5698-5705. DOI:10.1167/ iovs. 15-16708
Purpose. To identify eyes of patients with diabetes type 2 that show progression of retinal disease within a 1-year period using noninvasive techniques.

Methods. Three hundred seventy-four type 2 diabetic patients with mild nonproliferative diabetic retinopathy (Early Treatment Diabetic Retinopathy Study [ETDRS] level 20 or 35) were included in a 12-month prospective observational study to identify retinopathy progression. Four visits were scheduled at 0, 3, 6, and 12 months. Microaneurysm (MA) activity using the RetmarkerDR and retinal thickness using spectral-domain optical coherence tomography (SD-OCT) were assessed by a central reading center at all visits and ETDRS severity level in the first and last visits.

RESults. Three hundred thirty-one eyes/patients completed the study. Microaneurysm formation rate greater than or equal to 2 was present in $68.1 \%$ of the eyes and MA turnover greater than or equal to 6 in $54.0 \%$ at month 6 . Higher MA turnover values were registered in eyes that showed progression in ETDRS severity level $(P<0.03)$. There were also significant correlations between increased microaneurysm activity and increases in retinal thickness. Spectral-domain OCT identified clinical macular edema in 24 eyes/patients (6.7\%) and subclinical macular edema in 104 eyes/patients (28.9\%) at baseline. Progression of retinal thickening was registered in eyes that had either subclinical or clinical macular edema at baseline.

Conclusions. Changes in MA activity measured with RetmarkerDR and in central retinal thickness in eyes with mild nonproliferative diabetic retinopathy and diabetes type 2 are able to identify eyes at risk of progression. These eyes/patients should be selected for inclusion in future clinical trials of drugs targeted to prevent diabetic retinopathy progression to visionthreatening complications. (ClinicalTrials.gov number, NCT01145599.)

Keywords: diabetic retinopathy, biomarkers, microaneurysms, macular edema, diabetes type 2 
$\mathrm{D}$ iabetic retinopathy (DR) is a common and serious ophthalmic condition. It is the leading cause of blindness among working age adults in the United States. ${ }^{1}$ Disability consequent to vision loss related to eye disease among people with diabetes threatens independence and can lead to reduced quality of life. ${ }^{2}$

However, the rate of progression and risk for vision loss appears to vary widely between patients despite similar metabolic control. ${ }^{3}$

Therefore, the identification of different DR phenotypes characterized by different dominant retinal pathology and different rates of progression to vision-threatening retinopathy and vision loss including center-involved macular edema, is of major relevance for personalized management of DR and for clinical trial design and patient selection. ${ }^{3}$ The inclusion of eyes/patients in a clinical trial that do not progress during the period of the trial makes it impractical to enroll the required number of patients to detect differences in efficacy between the drug being tested and the placebo. ${ }^{4}$

It is therefore of great interest to identify and characterize eyes/patients that show disease activity and are at higher risk for developing vision-threatening retinopathy. The aim of this 1-year multicenter observational study was to identify progression in patients with mild nonproliferative DR (NPDR) by evaluating repeated noninvasive routine tests such as color fundus photography (CFP) and spectral-domain optical coherence tomography (SD-OCT).

\section{MeTHODS}

\section{Patients}

This was a prospective, multicenter, observational study, designed to follow eyes/patients with mild NPDR (20 and 35 of the Early Treatment Diabetic Retinopathy Study [ETDRS] classification) for a period of 1 year. The study was an investigator-driven observational study sponsored by the European Vision Institute Clinical Research Network (provided in the public domain by EVICR.net). ${ }^{5,6}$

A total of 374 patients were recruited between September 2010 and June 2012 in 19 European clinical sites and followed over a 1-year period. Men and women with diagnosed adultonset type 2 diabetes, age 35 to 82 years, mild NPDR (20 and 35 of ETDRS classification), best corrected visual acuity (BCVA) greater than or equal to 75 letters $(\geq 20 / 32)$ and refraction with spherical equivalent less than \pm 5 diopters (D) were included in the study. Exclusion criteria included the presence of cataract or other eye disease that may interfere with fundus examination, glaucoma, other retinal disease, any eye surgery within a period of 6 months, dilatation of the pupil less than 5 $\mathrm{mm}$ and previous or requiring treatment for DR at screening. All patients gave written consent. The tenets of the Declaration of Helsinki were followed and approval was obtained from each institutional review board (ClinicalTrials.gov Identifier: NCT01145599).

At baseline visit, patients' body weight, height, blood pressure, and concomitant medication were recorded. Based on the inclusion/exclusion criteria, one eye per patient was selected by the physician as the study eye. When both eyes fulfilled the same criteria, one of the eyes was selected by choosing sequentially the left or the right eye.

Patients performed four study visits: V0 (month 0), V3 (month 3), V6 (month 6), and V12 (month 12). At each visit, the study eyes underwent a complete ophthalmic examination, which included BCVA, slit-lamp examination, IOP measurements, digital fundus photography (CFP), and SD-OCT. Data on diabetes systemic medication, blood pressure measurements,
HbA1c levels, and body mass index (BMI) values were collected.

A central reading center (CORC-Coimbra Ophthalmology Reading Center, Portugal) classified the CFP for ETDRS grading and microaneurysms (MA) activity and the SD-OCT images.

During the period of the study and outside of the study visits, patients were followed in the clinical centers according to usual clinical practice. Identification of clinically significant macular edema (CSME) was based on ETDRS criteria. ${ }^{7,8}$

\section{Color Fundus Photography}

Color fundus photography was performed according to the ETDRS protocol at the first and last visits. The seven-field photographs were obtained at $30^{\circ} / 35^{\circ}$ for DR classification according to ETDRS grading scale. ${ }^{7}$ The grading of ETDRS levels was performed by double grading, with an intergrader reproducibility of $87 \%(\kappa=0.84)$ for sublevels of ETDRS and 93\% $(\kappa=0.91)$ for one step ETDRS level. Considering MAs and hard exudates, the more frequent alterations present in the eyes graded in this study, the intergrader reproducibility is $90 \%$ ( $\kappa=0.75)$ for MA and $83 \%(\kappa=0.71)$ for hard exudates. Additionally, $45^{\circ} / 50^{\circ}$ field- 2 color fundus images (macula) were taken at all visits for automated MA analysis, using an automated computer-aided diagnostic system (RetmarkerDR, Retmarker SA, Coimbra, Portugal) at the reading center.

RetmarkerDR is a patented computer software certified as a CE mark Class IIa medical device (European Union Certification of Conformity). The software was trained and tested prior to the CE medical device classification, that is, the algorithm (including the classifier) is objective and reproducible, it is not modified or retrained to any individual dataset.

This automated computer-aided diagnostic system consists of software earmarking MAs; it includes a coregistration algorithm that allows comparison within the same retinal location between different visits for the same eye.

RetmarkerDR calculates for each eye/patient the number of MA in each visit and the number of MAs that appear and/or disappear from one visit to the other, allowing calculation of the number of MA appearing and/or disappearing per time interval (i.e., the MA formation rate and the MA disappearance rate, respectively). The MA turnover is computed as the sum of the MA formation and MA disappearance rates. ${ }^{9-11}$

Previous work from our group ${ }^{9,12}$ showed a good intergrader agreement for the total number of MAs earmarked and the MA turnover for three independent human graders.

The RetmarkerDR shows a similar intergrader agreement for the total number of MAs, MA formation rate, and MA turnover (when compared with a human grader, intraclass correlation coefficients were 0.857 and 0.806 , respectively) while showing no intragrader variability as opposed to human graders, being, therefore, a reliable tool for MA assessment.

In this study, the MA formation rate and MA turnover were computed for all the eyes/patients at months 3, 6, and 12 (V3, V6, and V12, respectively). Patients were thereafter classified based on the presence of MA formation rate greater than or equal to 2, according to Nunes et al. ${ }^{12}$ and on the presence of a MA turnover greater than or equal to $6^{3}$, according to Nunes et al. ${ }^{3}$ An independent verification was also achieved by revisiting the CALDIRET data. ${ }^{11}$

These cut-off values have been proposed as predictive of progression of diabetic retinopathy. ${ }^{3,11,12}$

\section{Optical Coherence Tomography}

Spectral-domain OCT was performed using Cirrus ( $n=194$; Carl Zeiss Meditec, Inc., Dublin, CA, USA), Spectralis ( $n=166$; Heidelberg Engineering, Heidelberg, Germany), Copernicus ( $n$ 
= 4; Optopol, Zawiercie, Poland), or three-dimensional (3D) OCT-1000 ( $n=10$; Topcon Corporation, Tokyo, Japan).

Retinal thickness (RT) in the central subfield (central area with $1 \mathrm{~mm}$ in diameter), and the four areas of the inner and outer rings (within $1-3 \mathrm{~mm}$ and $3-6 \mathrm{~mm}$, respectively) were collected. Only Cirrus and Spectralis SD-OCT data are reported ( $n=360$ ), because of availability of normative data and the number of patients examined. The data from these instruments were analyzed independently.

To identify eyes/patients with increased RT in the central subfield (clinical and subclinical macular edema) and in the inner and outer rings, the reference values established by DRCR.net were used:

For clinical macular edema (ClinicalTrials.gov Identifier: NCT01909791) ${ }^{13}$ :

1. Retinal thickness greater than or equal to $290 \mu \mathrm{m}$ in women and greater than or equal to $305 \mu \mathrm{m}$ in men for Cirrus SD-OCT; and

2. Retinal thickness greater than or equal to $305 \mu \mathrm{m}$ in women and greater than or equal to $320 \mu \mathrm{m}$ in men for Spectralis SD-OCT.

For subclinical macular edema ${ }^{14-16}$ :

1. Retinal thickness greater than $260 \mu \mathrm{m}$ and less than 290 $\mu \mathrm{m}$ in women and greater than $275 \mu \mathrm{m}$ and less than $305 \mu \mathrm{m}$ in men, for Cirrus SD-OCT; and

2. Retinal thickness greater than $275 \mu \mathrm{m}$ and less than 305 $\mu \mathrm{m}$ in women and greater than $290 \mu \mathrm{m}$ and less than $320 \mu \mathrm{m}$ in men for Spectralis SD-OCT.

For the inner and outer rings (ClinicalTrials.gov Identifier: NCT01331005):

1. Normal RT, if there was no more than one area above normal range (normal mean $+2 \mathrm{SD}$ ) and no area $15 \mu \mathrm{m}$ above normal range; and

2. Increased RT, if there was at least two areas above normal range and/or one area $15 \mu \mathrm{m}$ above normal range.

The nine SD-OCT areas were classified as decreased, normal, or increased relatively to available Cirrus normative data. Because no normative database is available for Spectralis SD-OCT, a scaling factor was used based on analysis of 113 eyes/patients that performed both Cirrus and Spectralis SDOCT in the study. The RT values were higher with Spectralis SD-OCT: $+16.6 \mu \mathrm{m}$ for the central subfield, $+18.1 \mu \mathrm{m}$ for the inner ring, and $+17.7 \mu \mathrm{m}$ for the outer ring. Based on these differences, RT values of Spectralis SD-OCT were scaled to Cirrus SD-OCT considering $-18 \mu \mathrm{m}$ in inner and outer ring. For the central subfield the mean difference between Spectralis and Cirrus SD-OCT of $-15 \mu \mathrm{m}$ reported by the DRCR.net was taken into account.

\section{Sample Size}

To characterize retinal disease progression in type 2 diabetic patients with early NPDR a sample size minimum of 356 eyes/ patients was estimated based on the MA turnover results published by Nunes et al. ${ }^{12}$ for a statistical power of $90 \%$, an $\alpha$ level of 0.05 and a drop-out of $10 \%$ (i.e., considering that $60 \%$ of the patients with a MA formation rate $\geq 2$ will develop CSME in a 2 years' time interval and that only $20 \%$ of the patients with mild NPDR will have a MA formation rate $\geq 2$ ).

\section{Data Analysis}

Categorical variables are summarized with frequencies and percentages and numerical variables with mean and SD.
To test statistically significance differences at baseline between eyes/patients included and dropped out of the study a univariate analysis was performed with Bonferroni correction of the $P$ value. The $\chi^{2}$ test was used for categorical variables, and the Student's $t$-test or the nonparametric Mann-Whitney $U$ tests were used for continuous variables.

To compare the average evolution between visits of MA parameters (formation rate and turnover) and RT parameters the repeated measures ANOVA was performed.

To analyze associations between continuous variables the Pearson correlation was computed.

All statistical analyses were performed with STATA version 12.1 (StataCorp LP, College Station, TX, USA), and $P$ values less or equal than 0.05 were considered statistically significant results.

\section{Results}

From the 374 patients/eyes enrolled in the study (174 left eyes and 200 right eyes), 331 completed the study, that is, completed the 1-year follow-up period or developed CSME (ETDRS criteria) needing treatment (three eyes/patients). Two patients deceased between baseline and the last visit and 41 withdrew from the study (Fig.).

Baseline characteristics of patients enrolled in the study are presented in Table 1.

No statistically significant differences were found at baseline between the eyes/patients included in the study and the 43 eyes/patients that dropped out of the study.

Three eyes/patients were diagnosed with CSME during the 1-year follow-up period, all males, age ranging from 61 to 72 years and with HbA1c levels ranging from 5.9\% to $8.5 \%$. The ETDRS level was 35C for one eye/patient and 35D for the other 2. All three eyes had a MA formation rate at month 3 greater than or equal to 2 and a MA turnover greater than or equal to 6 . One of three patients had at baseline the OCT criteria for macular edema (central subfield $>305 \mu \mathrm{m}$; Cirrus SD-OCT).

Twenty three eyes/patients showed an increase in ETDRS level from screening visit to V12 and 24 patients showed a decrease in ETDRS level (Table 2).

\section{Microaneurysm Parameters}

Microaneurysm formation rate greater than or equal to 2 was present in 68.1\% [95\% CI: 63.0\%-73.1\%] of the eyes and MA turnover greater than or equal to 6 in $54.0 \%$ [95\% CI: $48.5 \%-$ $59.4 \%]$ at V6.

The MA formation rate and MA turnover at V6 showed a strong correlation with the values at V3 and V12. For the MA formation rate the correlation between V12 and V3 was $r=$ $0.950(P<0.001)$, and between V12 and V6 $r=0.912(P<$ $0.001)$. For the MA turnover the correlation between V12 and V3 was $r=0.957(P<0.001)$, and between V12 and V6 $r=$ $0.937(P<0.001)$.

The results show that there is a correlation between MA turnover values obtained at 6 and 12 months and changes in ETDRS level.

For the 23 eyes/patients that showed an increase in ETDRS level from baseline to $\mathrm{V} 12,39.1 \%$ had a MA turnover greater than or equal to 6 at V6, whereas only $8.3 \%$ of the eyes/patients that showed a decrease in ETDRS level had a MA turnover greater than or equal to $6(P=0.013)$. At V12, 34.8\% of the 23 eyes/patients that showed an increase in ETDRS level from baseline to V12, had a MA turnover greater than or equal to 6 , whereas only $8.3 \%$ of the eyes/patients that showed a decrease in ETDRS level had a MA turnover greater than or equal to $6(P$ $=0.027$; Table 3 ). 


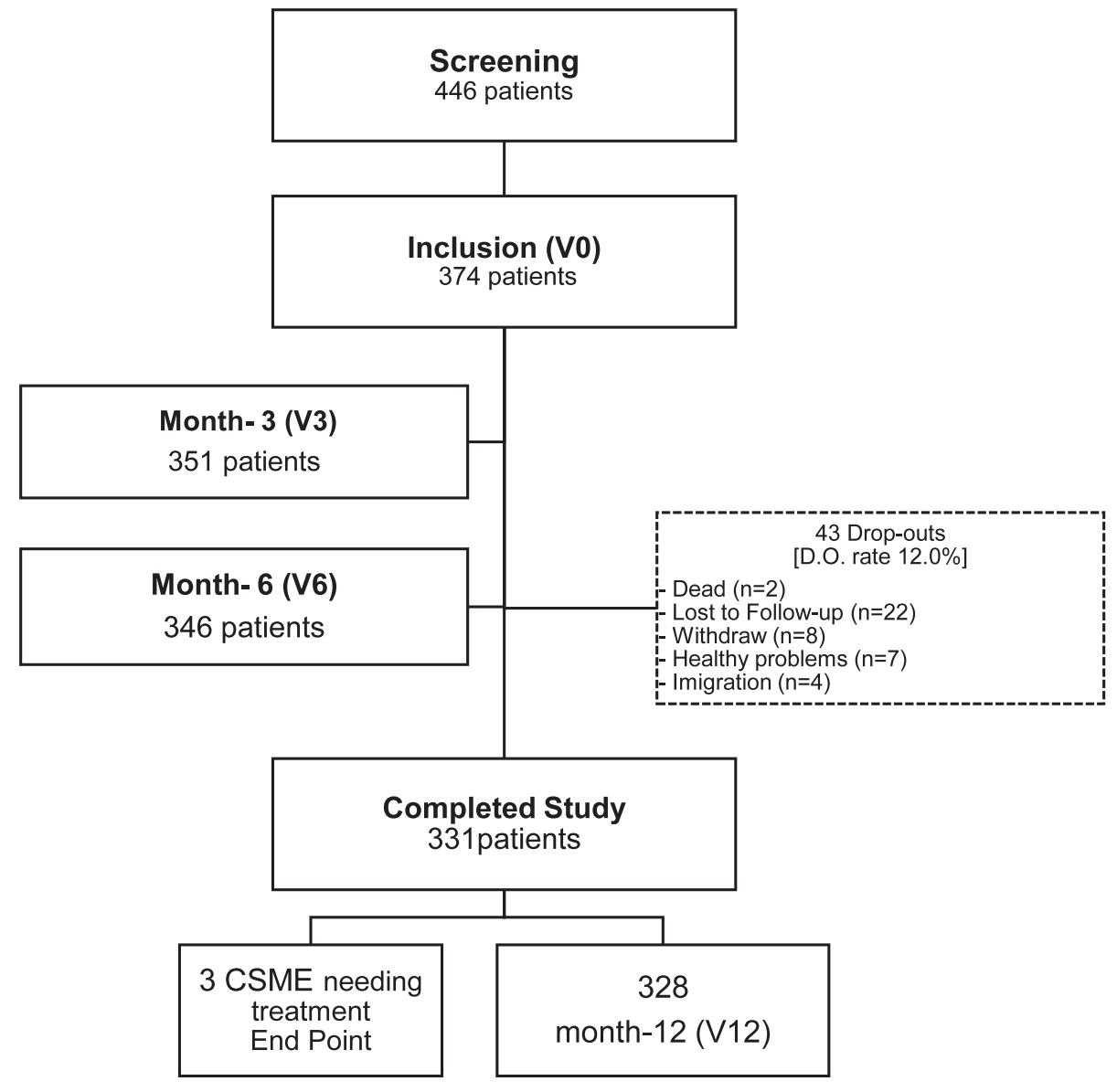

Figure. Consort flow chart.

The MA parameters showed very weak, positive statistically significant correlations with HbA1c levels and BMI $(r<0.181)$.

There was a very weak, positive statistically significant correlation at V6 between MA parameters (number, formation rate, and turnover) and the $\mathrm{RT}$ in the central subfield ( $r=$ $0.141, r=0.170$, and $r=0.156$, respectively). For the inner and outer ring areas statistically significant weak correlations were also found for most of the respective areas and visits (for the inner ring $r<0.193$ and for the outer ring $r<0.328$ ).

Associations were found between the MA formation rate and MA turnover cut-off values ( $\geq 2$ and $\geq 6$, respectively) and the presence of increased $\mathrm{RT}$ in the central subfield, inner and/ or outer ring:

1. Of the eyes/patients with an increased RT in the central subfield, inner and/or outer ring, 61.8\% (95\% CI: 44.6\%$79.0 \%$ ) to $76.7 \%$ (95\% CI: $65.6 \%-87.7 \%$ ) showed an MA formation rate greater than or equal to 2 and/or an MA turnover greater than or equal to 6 ; and

2. Of the eyes/patients with a MA formation rate less than 2 and/or a MA turnover less than 6, 81.3\% (95\% CI: $74.8 \%-$ $87.8 \%$ ) to $87.7 \%$ (95\% CI: $82.3 \%-90.1 \%$ ) showed a normal $\mathrm{RT}$ in the central subfield, inner and/or outer ring.

Table 1. Baseline Characteristics of the Eyes/Patients That Were Included and Dropped Out of the Study

\begin{tabular}{|c|c|c|c|}
\hline & $\begin{array}{l}\text { Patients Included in the Study, } \\
\qquad n=374\end{array}$ & $\begin{array}{l}\text { Drop-Out Patients, } \\
\qquad n=43\end{array}$ & $\boldsymbol{P}$ \\
\hline Sex, female/male, frequency (\%) & $131(35.0) / 243(65.0)$ & $19(44.2) / 24(55.8)$ & 0.24 \\
\hline \multicolumn{4}{|l|}{ ETDRS level } \\
\hline 20 , frequency $(\%)$ & $70(18.7)$ & $12(27.9)$ & 0.15 \\
\hline 35 , frequency (\%) & $304(81.3)$ & $31(72.1)$ & \\
\hline Age, mean (SD), y & $60.3(9.0)$ & $58.3(9.89)$ & 0.17 \\
\hline HbA1c, mean (SD), \% & $7.8(1.5)$ & $7.8(1.5)$ & 0.83 \\
\hline Systolic blood pressure, mean (SD), mm Hg & $137.6(16.6)$ & $135.6(17.8)$ & 0.46 \\
\hline Diastolic blood pressure, mean (SD), $\mathrm{mm} \mathrm{Hg}$ & $77.4(10.0)$ & $81.1(8.0)$ & $0.02 *$ \\
\hline BMI, mean (SD), $\mathrm{kg} / \mathrm{m}^{2}$ & $29.9(5.4)$ & $30.6(5.4)$ & 0.40 \\
\hline BCVA, mean (SD), letters & $85.3(4.6)$ & $84.1(5.0)$ & 0.11 \\
\hline No. of MAs, mean (SD) & $3.7(5.4)$ & $2.5(3.5)$ & 0.16 \\
\hline
\end{tabular}

\footnotetext{
* Bonferroni corrected $P$ value 0.006 .
} 
TABLE 2. Number of Eyes/Patients With ETDRS Changes From Vo to V12

\begin{tabular}{|c|c|c|c|c|c|c|c|}
\hline \multirow{2}{*}{$\begin{array}{c}\text { ETDRS } \\
\text { Level at V0 }\end{array}$} & \multicolumn{6}{|c|}{ ETDRS Level at V12 } & \multirow[b]{2}{*}{ Total } \\
\hline & 10 & 15 & 20 & 35 & 43 & 47 & \\
\hline 20 & 1 & 1 & 0 & 19 & 0 & 0 & 21 \\
\hline 35 & 2 & 0 & 20 & 0 & 3 & 1 & 26 \\
\hline Total & 3 & 1 & 20 & 19 & 3 & 1 & 47 \\
\hline
\end{tabular}

Finally, MA parameters obtained at V6 were the most informative, indicating that the time interval of 6 months is necessary to evaluate MA activity.

\section{Retinal Thickness}

Considering results obtained at baseline with the Cirrus and the Spectralis and based on their respective normal database, an increased RT in the central subfield was found in $9.8 \%(95 \%$ CI: 5.6\%-13.9\%) when using the Cirrus and 10.8\% (95\% CI: $6.1 \%-15.6 \%$ ) using Spectralis for a total of $10.3 \%$ of the eyes examined, while a decreased RT was found in $1.5 \%(95 \% \mathrm{CI}$ : 0\%-3.3\%) using the Cirrus and 9.6\% (95\% CI: 5.1\%-14.1\%) using Spectralis for a total of $5.3 \%$ of the eyes (Table 4). In the inner ring areas, this proportion was maintained. The highest number of eyes showing increased RT was found in the outer ring areas $(13.3 \%-23.1 \%)$.

In the central subfield 24 eyes/patients (6.7\%, corresponding to $6.2 \%$ [95\% CI: $2.8 \%-9.6 \%]$ with the Cirrus and $7.2 \%$ [95\% CI: 3.3\%-11.2\%] with the Spectralis) had a OCT diagnosis of clinical macular edema, according to the DRCR.net standards (Table 4). ${ }^{13}$ Subclinical macular edema was identified in 104 eyes/patients (28.9\%, corresponding to $32.0 \%$ [95\% CI: $25.4 \%$ $38.5 \%$ ] with the Cirrus and $25.3 \%$ [95\% CI: 18.7\%-31.9\%] with the Spectralis; Table 4).

Eyes/patients classified as subclinical and clinical macular at baseline occurred in eyes/patients with ETDRS level 20 and 35, respectively $8.3 \%$ and $25.9 \%$.

No correlation was found between the eyes/patients that showed an increase in ETDRS level at the end of 1 year of follow-up and the eyes/patients that presented higher retinal thickness values in the central subfield (i.e., subclinical and clinical macular edema; $P=0.61$ ).

The eyes that showed increases in RT were in general the same eyes during the entire follow-up period. Eighty-two percent $(82.9 \%)$ of the eyes with increased RT in the central subfield at baseline had increased RT at V12. Increase in RT occurred also in the inner ring (73.2\%) and in the outer ring (72.8\%).

Increase in central subfield RT over the 1-year follow-up was registered in eyes that had subclinical and clinical macular edema at baseline. In the eyes with clinical macular edema at baseline the mean RT increase was $4.8 \mu \mathrm{m}$ (95\% CI: $-3.3 \mu \mathrm{m}$ to $12.9 \mu \mathrm{m})$ for Cirrus SD-OCT and $7.63 \mu \mathrm{m}(95 \% \mathrm{CI}:-25.5 \mu \mathrm{m}$ to $40.8 \mu \mathrm{m})$ for Spectralis SD-OCT.

\section{Discussion}

This 1-year, observational, longitudinal study of 374 patients with diabetes type 2, mild NPDR (ETDRS levels 20 and 35) and good visual acuity, examined at baseline and at 3, 6, and 12 months, by 19 clinical sites across Europe, showed that changes in RT and MA activity vary widely between patients with similar metabolic control, duration of disease and ETDRS retinopathy severity level, confirming previous reports. ${ }^{3,12}$

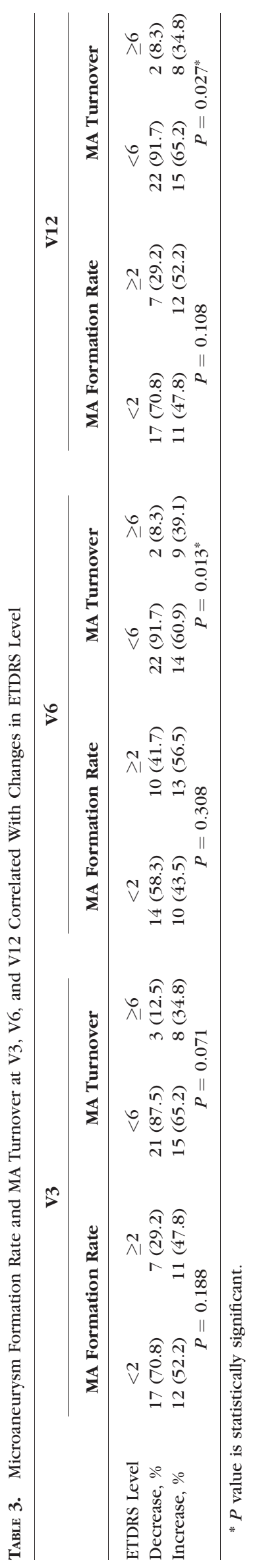


TABLE 4. Frequency (Number and Percentage) of the Eyes/Patients With Decreased/Normal/Increased RT at Baseline

\begin{tabular}{|c|c|c|}
\hline & $\begin{array}{c}\text { Cirrus, } \\
n=194\end{array}$ & $\begin{array}{c}\text { Spectralis, } \\
n=166\end{array}$ \\
\hline \multicolumn{3}{|l|}{ Central subfield } \\
\hline Decreased (mean - $2 \mathrm{SD})$ & $3(1.5)$ & $16(9.6)$ \\
\hline Normal & $172(88.7)$ & $132(79.5)$ \\
\hline Increased (mean + 2 SD) & $19(9.8)$ & $18(10.8)$ \\
\hline \multicolumn{3}{|l|}{ Inner ring } \\
\hline \multicolumn{3}{|l|}{ Nasal } \\
\hline Decreased (mean - $2 \mathrm{SD}$ ) & $7(3.6)$ & $28(16.9)$ \\
\hline Normal & $168(86.6)$ & $124(74.7)$ \\
\hline Increased (mean + $2 \mathrm{SD})$ & $19(9.8)$ & $14(8.4)$ \\
\hline \multicolumn{3}{|l|}{$\rightarrow$ Increased (mean $+2 \mathrm{SD}$} \\
\hline$+15 \mu \mathrm{m})$ & $\rightarrow 5$ (2.6) & $\rightarrow 2(1.2)$ \\
\hline \multicolumn{3}{|l|}{ Superior } \\
\hline Decreased (mean - 2 SD) & $5(2.6)$ & $26(15.7)$ \\
\hline Normal & $168(86.6)$ & $123(74.1)$ \\
\hline $\begin{array}{l}\text { Increased (mean }+2 \mathrm{SD}) \\
\rightarrow \text { Increased (mean }+2 \mathrm{SD}\end{array}$ & $21(10.8)$ & $17(10.2)$ \\
\hline$+15 \mu \mathrm{m})$ & $\rightarrow 4(2.1)$ & $\rightarrow 1(0.6)$ \\
\hline \multicolumn{3}{|l|}{ Temporal } \\
\hline Decreased (mean $-2 \mathrm{SD})$ & $7(3.6)$ & $26(15.7)$ \\
\hline Normal & $167(86.1)$ & $118(71.1)$ \\
\hline Increased (mean + 2 SD) & $20(10.3)$ & $22(13.3)$ \\
\hline $\begin{array}{l}\rightarrow \text { Increased (mean }+2 \mathrm{SD} \\
\quad+15 \mu \mathrm{m})\end{array}$ & $\rightarrow 5$ (2.6) & $\rightarrow 4$ (2.4) \\
\hline \multicolumn{3}{|l|}{ Inferior } \\
\hline Decreased (mean $-2 \mathrm{SD})$ & $10(5.2)$ & $28(16.9)$ \\
\hline Normal & $163(84)$ & $126(75.9)$ \\
\hline $\begin{array}{l}\text { Increased (mean }+2 \mathrm{SD}) \\
\rightarrow \text { Increased }(\text { mean }+2 \mathrm{SD}\end{array}$ & $21(10.8)$ & $12(7.2)$ \\
\hline$+15 \mu \mathrm{m})$ & $\rightarrow 5$ (2.6) & $\rightarrow 2(1.2)$ \\
\hline
\end{tabular}

Outer ring

Nasal

Decreased (mean - $2 \mathrm{SD}$ )

Normal

Increased (mean $+2 \mathrm{SD})$

$\rightarrow$ Increased (mean +2 SD

$+15 \mu \mathrm{m})$

Superior

Decreased (mean $-2 \mathrm{SD})$

Normal

Increased (mean $+2 \mathrm{SD})$

$\rightarrow$ Increased (mean +2 SD

$+15 \mu \mathrm{m})$

Temporal

Decreased (mean - $2 \mathrm{SD}$ )

Normal

Increased (mean $+2 \mathrm{SD})$

$\rightarrow$ Increased (mean +2 SD

$+15 \mu \mathrm{m})$

Inferior

Decreased (mean - $2 \mathrm{SD}$ )

Normal

Increased (mean $+2 \mathrm{SD})$

$\rightarrow$ Increased (mean +2 SD

$+15 \mu \mathrm{m})$

$6(3.1)$

$13(7.8)$

$158(81.4) \quad 135(81.3)$

$30(15.5) \quad 18(10.8)$

$\rightarrow 10(5.2) \quad \rightarrow 0(0.0)$

9 (4.6)

$158(81.4)$

$9(5.5)$

$120(72.7)$

27 (13.9) $\quad 36(21.8)$

$\rightarrow 7$ (3.6) $\quad \rightarrow 0(0.0)$

$4(2.1)$

$160(82.5)$

7 (4.2)

$30(15.5) \quad 53(31.9)$

$\rightarrow 10(5.2) \quad \rightarrow 2(1.2)$

8 (4.1)

$162(83.5)$

$9(5.4)$

$24(12.4) \quad 39(23.5)$

$\rightarrow 6(3.1) \quad \rightarrow 0(0.0)$
Table 4. Continued

\begin{tabular}{|c|c|c|}
\hline & $\begin{array}{l}\text { Cirrus, } \\
n=194\end{array}$ & $\begin{array}{l}\text { Spectralis, } \\
n=166\end{array}$ \\
\hline \multicolumn{3}{|l|}{ Central subfield } \\
\hline Subclinical macular edema & $62(32.0)$ & $42(25.3)$ \\
\hline Clinical macular edema & $12(6.2)$ & $12(7.2)$ \\
\hline Inner ring increased & 23 (11.9) & $19(11.5)$ \\
\hline Outer ring increased & $36(18.6)$ & $40(24.1)$ \\
\hline \multicolumn{3}{|l|}{$\begin{array}{l}\text { 1-year change (eyes/patients that } \\
\text { completed the study) }\end{array}$} \\
\hline $\begin{array}{l}\text { Subclinical to clinical macular } \\
\text { edema }\end{array}$ & 5 of 52 & 7 of 38 \\
\hline $\begin{array}{l}\text { Clinical to subclinical macular } \\
\text { edema }\end{array}$ & 2 of 10 & 4 of 11 \\
\hline
\end{tabular}

Higher MA turnover values at V6 were found to be associated with increase in ETDRS level at the end of the 1 year of follow-up, which supports previous reports that MA turnover can identify eyes likely to progress. ${ }^{3,12}$

Regarding RT changes, it is of relevance that of the 360 eyes/patients (considering only those examined with Cirrus or Spectralis SD-OCT) included in the study, center-involving macular edema with indication for treatment based on OCT values, ${ }^{13}$ was found to be present in 24 eyes (6.7\%). These eyes were not diagnosed on slit-lamp examination. There were also 104 eyes $(28.9 \%)$ that met the OCT criteria for subclinical macular edema. ${ }^{14-16}$

Microaneurysms parameters (number, formation rate, and turnover) correlated with RT increases, with the strongest correlations found with MA formation rate and MA turnover. Automated image analysis of MA turnover is shown, in this study, to identify retinal vascular disease activity at this retinopathy stage, predicting ETDRS level changes and showing also, correlations with increased RT (i.e., macular edema).

It is also of note that when considering all the patients included in the study, there was little progression in RT values over the 1-year follow-up period. Progression was only found in the eyes that already had thickness increases at baseline and mainly in the central subfield. It is also noteworthy that the changes identified in RT at baseline remained generally in the same location during the entire period of follow-up. These findings show that 1 year is a relatively short time interval for a clinical trial testing therapies against increases in RT (i.e., retinal edema) unless only patients with increased RT in the central subfield are selected for the trial. However, our observations showed that RT measurements performed with SD-OCT are reliable and may be followed accurately, and that eyes with subclinical macular edema at baseline are good candidates for clinical trials.

It is of interest that no significant correlation was found between the presence of increased $\mathrm{RT}$ in the central subfield and ETDRS levels 20 or 35.

There have been a number of reports in the literature, suggesting that a decrease in RT attributed to a neurodegenerative process is a frequent finding in the early stages of diabetic retinal disease. ${ }^{17,18}$ This observation is not confirmed in our study. The number of eyes with abnormally increased RT almost doubled the number of eyes with decreased RT.

This multicenter, multinational study demonstrated that noninvasive procedures such as fundus photography and SDOCT are useful tools to monitor microvascular disease activity and development of edema in the early stages of diabetes (mild NPDR, ETDRS levels 20 and 35). The findings here described 
contribute to improve our understanding of DR progression and to improve the design of clinical trials aiming to test the effect of drugs to prevent DR progression to vision-threatening complications.

In conclusion, changes in MA activity measured with RetmarkerDR on fundus photographies and in central RT measured by SD-OCT show wide variations between different patients with mild NPDR and diabetes type 2 . It is possible, using these noninvasive procedures, SD-OCT and fundus photography, to identify the eyes that are at higher risk of progression. These eyes/patients should be the ones selected for inclusion in future clinical trials of drugs targeted to prevent DR progression.

\section{Acknowledgments}

The authors thank the EVICR.net Study Group. The centers and personnel involved in the study are listed in the Appendix. This study was an Investigator-Driven observational nonfunded study sponsored by EVICR.net.

Disclosure: L. Ribeiro, None; F. Bandello, Allergan (C), Novartis (C), Farmila-Thea (C), Bayer Schering Pharma (C), Pfizer (C), Alcon (C), Bausch and Lomb (C), Genentech (C), Alimera Sciences (C), Sanofi-Aventis (C), Thrombogenics (C), Hoffmann-La Roche (C), Novagali Pharma (C); A.N. Tejerina, None; S. Vujosevic, None; M. Varano, Allergan (C), Novartis (C), Bayer (C); C. Egan, None; S. Sivaprasad, Allergan (C), Bayer (C), Novartis (C), Roche (C); G. Menon, None; P. Massin, None; F.D. Verbraak, None; H. LundAndersen, None; J.P. Martinez, None; I. Jürgens, None; E. Smets, None; C. Coriat, None; P. Wiedemann, None; V. Ágoas, None; G. Querques, Alcon (C), Allergan (C), Alimera (C), Bayer (C), Baush and Lomb (C), Novartis (C), Ophthotech (C); F.G. Holz, Acucela (C), Alcon (C), Allergan (C), Bayer Healthcare (C), Genentech (C), Heidelberg Engineering (C), Novartis (C), Roche (C); S. Nunes, None; C. Neves, None; J. Cunha-Vaz, Alimera Sciences (C), Allergan (C), Bayer (C), Fovea Pharmaceuticals (C), GeneSignal (C), Novartis (C), OM Pharma (C), Pfizer (C), Roche (C), Zeiss (C)

\section{References}

1. Fong DS, Aiello L, Gardner TW, et al. Retinopathy in diabetes. Diabetes Care. 2004;27(suppl 1):S84-S87.

2. Sinclair AJ, Bayer AJ, Girling AJ, Woodhouse KW. Older adults, diabetes mellitus and visual acuity: a community-based casecontrol study. Age Ageing. 2000;29:335-339.

3. Nunes S, Ribeiro L, Lobo C, Cunha-Vaz J. Three different phenotypes of mild nonproliferative diabetic retinopathy with different risks for development of clinically significant macular edema. Invest Ophthalmol Vis Sci. 2013;54:4595-4604.

4. Aiello LP, Vignati L, Sheetz MJ, et al. Oral protein kinase c $\beta$ inhibition using ruboxistaurin: efficacy, safety, and causes of vision loss among 813 patients (1,392 eyes) with diabetic retinopathy in the Protein Kinase $\mathrm{C} \beta$ Inhibitor-Diabetic Retinopathy Study and the Protein Kinase C $\beta$ InhibitorDiabetic Retinopathy Study 2. Retina. 2011;31:2084-2094.

5. Cunha-Vaz J, Martinho C. Developing an international network for clinical research in ophthalmology: the European Vision Institute Clinical Research Network (EVICR.net). Clin Investig (Lond). 2011;1:375-380.

6. EVICR: European Network of Clinical Research in Ophthalmology Information Update - November 2012. Ophthalmologica. 2014;231:180-184.

7. Grading diabetic retinopathy from stereoscopic color fundus photographs-an extension of the modified Airlie House classification. ETDRS report number 10. Early Treatment
Diabetic Retinopathy Study Research Group. Ophthalmology. 1991;98(5 suppl):786-806.

8. Treatment techniques and clinical guidelines for photocoagulation of diabetic macular edema. Early Treatment Diabetic Retinopathy Study Report Number 2. Early Treatment Diabetic Retinopathy Study Research Group. Ophthalmology. 1987;94: 761-774.

9. Bernardes R, Nunes S, Pereira I, et al. Computer-assisted microaneurysm turnover in the early stages of diabetic retinopathy. Ophthalmologica. 2009;223:284-291.

10. Ribeiro ML, Nunes S, Cunha-Vaz J. Microaneurysm turnover at the macula predicts risk of development of clinically significant macular edema in persons with mild nonproliferative diabetic retinopathy. Diabetes Care. 2013;36:1254-1259.

11. Haritoglou C, Kernt M, Neubauer A, et al. Microaneurysm formation rate as a predictive marker for progression to clinically significant macular edema in nonproliferative diabetic retinopathy. Retina. 2014;34:157-164.

12. Nunes S, Pires I, Rosa A, Duarte L, Bernardes R, Cunha-Vaz J. Microaneurysm turnover is a biomarker for diabetic retinopathy progression to clinically significant macular edema: findings for type 2 diabetics with nonproliferative retinopathy. Ophthalmologica. 2009;223:292-297.

13. Chalam KV, Bressler SB, Edwards AR, et al. Retinal thickness in people with diabetes and minimal or no diabetic retinopathy: Heidelberg Spectralis optical coherence tomography. Invest Ophthalmol Vis Sci. 2012;53:8154-8161.

14. Bressler NM, Miller KM, Beck RW, et al. Observational study of subclinical diabetic macular edema. Eye (Lond). 2012;26:833840.

15. Browning DJ, Fraser CM. The predictive value of patient and eye characteristics on the course of subclinical diabetic macular edema. Am J Ophthalmol. 2008;145:149-154.

16. Pires I, Santos AR, Nunes S, Lobo C, Cunha-Vaz J. Subclinical macular edema as a predictor of progression to clinically significant macular edema in type 2 diabetes. Ophthalmologica. 2013;230:201-206.

17. Asefzadeh B, Fisch BM, Parenteau CE, Cavallerano AA. Macular thickness and systemic markers for diabetes in individuals with no or mild diabetic retinopathy. Clin Experiment Ophthalmol. 2008;36:455-463.

18. Van Dijk HW, Kok PHB, Garvin M, et al. Selective loss of inner retinal layer thickness in type 1 diabetic patients with minimal diabetic retinopathy. Invest Ophthalmol Vis Sci. 2009;50: 3404-3409.

\section{ApPendix}

\section{Protocol ECR-RET-2010-02 Study Group}

AIBILI-Clinical Trial Centre (CS01), Coimbra, Portugal: principal investigator: Luísa Ribeiro, MD, MSc; investigators: João Figueira, MD, MSc; Isabel Pires, MD; Sérgio Leal, MD; other study personnel: Silvia Simão, BSc; Ana Rita Santos, MSc; and Isabel Simões.Centre Hospitalier Intercommunal de Creteil, University Paris Est Creteil (CSO3), Creteil, France: principal investigator: Giuseppe Querques, $\mathrm{MD}, \mathrm{PhD}$; investigator: Eric $\mathrm{H}$ Souied, MD, PhD Centre d'Investigation Clinique, Centre National d'Ophtalmologie des Quinze-Vingts (CS06), Paris, France: principal investigator: Caroline Coriat, MD Moorfields Eye Hospital, NHS Foundation Trust, NIHR Moorfields Clinical Research Facility (CS10), London, United Kingdom: principal investigator: Catherine Egan, MD, FRANZO; other study personnel: Hayley Boston; Simona Degli Esposti; and Vincent 
Rocco. Antwerp University Hospital (CS12), Antwerp, Belgium: principal investigator: Erica Smets, MD; other study personnel: Danny G.P. Mathysen, PhD Hôpital Lariboisière (CS14), Paris, France: principal investigator: Pascale Massin, MD, PhD; investigator: Ali Erginay, MD University of Bonn (CS15), Bonn, Germany: principal investigator: Frank G. Holz, MD; investigator: Monika Fleckenstein, MD G.B. Bietti Eye Foundation IRCCS (CS20), Rome, Italy: principal investigator: Monica Varano, MD; investigators: Andrea Cacciamani, MD; Mariacristina Parravano, MD; and Pamela Cosimi, MD Academic Medical Center (CS25), Amsterdam, Netherlands: principal investigator: Frank Verbraak, MD, PhD; investigators: M. Mourits, MD, PhD; and R. O. Schlingemann, MD, PhD; other study personnel: M. Wezel; C. Jansen-Kok; A. Althoff; D. de Vries; and M. Stam. University Eye Hospital Leipzig (CS27), Leipzig, Germany: principal investigator: Peter Wiedemann, $\mathrm{MD}, \mathrm{PhD}$; investigators: Claudia Jochmann, MD; and Christian Koch, MD; other study personnel: Daniela Vollhardt; and Christina Tilgner. Instituto de Oftalmologia Dr Gama Pinto (CS28), Lisbon, Portugal: principal investigator: Victor Ágoas, MD; other study personnel: Pedro Camacho, BSc. Glostrup Hospital,

Copenhagen University (CS30), Copenhagen, Denmark: principal investigator: Henrik Lund-Andersen, MD Institut Català de Retina (ICR) (CS38), Barcelona, Spain: principal investigator: Ignasi Jürgens, $\mathrm{MD}, \mathrm{PhD}$; investigators: Daniel Vila, MD; Antonio Morilla, PhD; Lluis Gonzalez, OD; Victor Martínez, OD; Gemma Pedrós, OD; Mireia García, OD; Yolanda Aradilla, OD; Alexia Martínez, OD; and Raimon Escudé, OD. Universita di Padova, Center for Clinical Trials (CS39), Padova, Italy: principal investigator: Stela Vujosevic, $\mathrm{MD}, \mathrm{PhD}$; investigators: Edoardo Midena, MD, PhD; Ferdinando Martini, MD; Francesca Urban, MD; and Anna Rita Daniele, MD Rotterdam
Eye Hospital (CS40), Rotterdam, Netherlands: principal investigator: Jose Martinez, MD Fundación para la Investigacion Biomedica y Sanitaria (FISABIO) (CS51), Valencia, Spain: principal investigator: Amparo Navea Tejerina, $\mathrm{MD}, \mathrm{PhD}$; investigators: Ester Fernandez-Lopez, MD; Adriana Fandiño, MD; M Carmen, MD; and Desco Esteban, MD; other study personnel: Pablo Perez Jord. Frimley Park Hospital Foundation Trust (CS66), Frimley, United Kingdom: principal investigator: Geeta Menon, MD, FRCS; investigators: Manju Chandran, MRCOphth, DNB, DO, MBBS; and Lorraine North, MSc, BSc; other study personnel: Deana Robson; and Maheswari Chekuri. University Vita Salute - Scientific Institute of San Raffael (CS67), Milan, Italy: principal investigator: Francesco Bandello, MD; investigators: Rosangela Lattanzio, MD; and Claudia Del Turco, MD; other study personnel: Giorgio Alto; Alessio Buzzotta; Marta Marelli, MSc; and Antonella Ribecca. Laser and Retinal Research Unit, King's Health Partners (CS69), London, United Kingdom: principal investigator: Sobha Sivaprasad, MD, FRCS; investigators: Roxanne Crosby-Nwaobi, MD

Reading Center: Coimbra Ophthalmology Reading Center: Christian Schwartz; Silvia Simão, BSc; Catarina Neves; Rui Pita; Luisa Ribeiro, MD, MSc; Ana Rita Santos, MSc; and Joana Ecsodi Study Coordinating Center: Coimbra Coordinating Centre for Clinical Research: Sandrina Nunes, MSc; and Sónia Simões, BSc

Statistical analysis: Coimbra Coordinating Centre for Clinical Research: Sandrina Nunes, MSc and Miguel Costa, MSc

EVICR.net Coordinating Centre: José Cunha-Vaz, MD, PhD; Cecília Martinho, BSc; and Rita Fernandes, BSc.

See EVICR.net Study Group (in the public domain) at http:// www.evicr.net/downloads/file270_gb.pdf. 